\title{
Copolimerização em Emulsão de Acetato de Vinila e Acrilato de Butila com Alto Teor de Sólidos
}

\author{
Mauri Palma \\ Faculdade de Ciências Farmacêuticas, Escola Politécnica, USP \\ Reinaldo Giudici \\ Departamento de Engenharia Química, Escola Politécnica, USP
}

\begin{abstract}
Resumo: Neste trabalho foi investigada a influência da concentração de uma mistura dos emulsificantes nonilfenol etoxilado e sulfato sódico de nonilfenol etoxilado (não iônico e iônico, respectivamente) e concentração de protetor coloidal, poli (álcool vinílico) na estabilidade coloidal de látices do copolímero acetato de vinila (AV)/acrilato de butila (AB) com alto teor de sólidos. Foram determinados o teor de sólidos e o tamanho médio das partículas ao longo da reação e foram obtidas as viscosidades das emulsões finais. Os ensaios foram realizados de acordo com um projeto fatorial com 3 níveis de concentração de emulsificantes e de protetor coloidal. Foi verificado que são necessárias maiores quantidades relativas de emulsificantes e de protetor coloidal pela massa total de monômeros para se obter látices estáveis com teores de sólidos de 70 e $73 \%(\mathrm{~m} / \mathrm{m})$. Os tamanhos médios das partículas no final de cada ensaio foram relativamente elevados (800 a $1000 \mathrm{~nm})$ e as viscosidades a $20^{\circ} \mathrm{C}$ relativamente baixas $(500 \mathrm{mPa}$.s $)$ para teor de sólidos de $67 \%(\mathrm{~m} / \mathrm{m})$; para teor de sólidos de $70 \%(\mathrm{~m} / \mathrm{m})$ as viscosidades variaram de 5.000 a $10.000 \mathrm{mPa} . \mathrm{s}$ e para teor de sólidos de $73 \%(\mathrm{~m} / \mathrm{m})$ a viscosidade foi de $365.000 \mathrm{mPa} . \mathrm{s}$
\end{abstract}

Palavras-chave: Alto teor de sólidos, copolimerização, acrilato de butila, acetato de vinila, polimerização em emulsão, reator semi contínuo.

\section{High Solid Contents Semi-Batch Emulsion Copolymerization of Vinyl Acetate and Butyl Acrylate}

Abstract: In this work the influence of the concentration of ionic (alkyl phenol polyglycol ether sodium sulfate), and nonionic (alkyl phenol polyglycol ether), surfactants mixture and of protective colloid (polyvinyl alcohol) on the colloidal stability of high solid contents butyl acrylate/vinyl acetate copolymers latexes was investigated. Solid contents and particle diameter were determined along the reaction and, at the end of each run, also the emulsion viscosity. The experiments were carried out according to a factorial design with three levels of surfactant and protective colloid concentrations. Higher amounts of surfactant and protective colloid per total monomers were required for the colloidal stability of emulsions with total solid contents of 70 and 73 wt. (\%). The particle diameters at the end of the experiment were rather large (800 to $1000 \mathrm{~nm}$ ) and the viscosities at $20{ }^{\circ} \mathrm{C}$ rather small (500 mPa.s) for solid contents of $67 \mathrm{wt}$. (\%); for higher values of solid contents (70 and 73 wt. (\%)) the viscosities were as high as 5000 to 10000 and $365000 \mathrm{mPa}$.s, respectively.

Keywords: High solid contents, copolymerization, vinyl acetate, emulsion polymerization, semi-batch.

\section{Introdução}

São várias as vantagens que sistemas com alto teor de sólidos podem apresentar. Dentre elas podem ser citadas: o menor custo de produção, armazenagem e de transporte e a não necessidade de concentrar a emulsão para algumas aplicações, como por exemplo, calafetações ${ }^{[1]}$. Além disso, emulsões com alto teor de sólidos apresentam um tempo menor de secagem e de formação de filmes. Do ponto de vista ambiental, no âmbito da Pollution Prevention e Green Technology, também é muito interessante a produção de emulsões com alto teor de sólidos, devido ao menor consumo de água durante a produção.
Em vista disso é crescente a demanda na indústria por emulsões com alto teor de sólidos ${ }^{[2-7]}$. Nesta condição surgem diversas dificuldades de processo a serem resolvidas, como, por exemplo: o aumento da viscosidade dificulta a homogeneização do meio e pode provocar incrustação, o que causaria paradas para limpeza do reator e perdas de produto; dificuldade do controle da temperatura devido à maior exotermia do que nos processos com baixo teor de sólidos e à maior dificuldade de transferência de calor em meios viscosos. Apesar da disponibilidade no mercado de látices com conteúdos superiores a $60 \%(\mathrm{~m} / \mathrm{m})$, poucos são os casos em que seja possível alcançar teores superiores a $55 \%(\mathrm{~m} / \mathrm{m}) \mathrm{em}$ reatores industriais sem o emprego de técnicas de concentra-

Autor para correspondência: Mauri Palma, Faculdade de Ciências Farmacêuticas, Escola Politécnica, USP, Av. Prof. Lineu Prestes 580, Bloco 16, CEP: 05508-000, São Paulo, SP, Brasil. E-mail: msapalma@usp.br 
ção pós-reação. Embora o mercado demande por látices com teor de sólidos superiores a $65 \%(\mathrm{~m} / \mathrm{m})$, com alta estabilidade coloidal, a pesquisa acadêmica ainda é escassa. Os trabalhos publicados que tratam do potencial da técnica de polimerização para a produção de látices concentrados utilizam usualmente estratégias fundamentalmente empíricas ${ }^{[2,7,8]}$. A viscosidade deve ser adequada, tanto durante a produção, quanto durante a preparação de produtos finais (por exemplo, tintas).

Um dos passos decisivos para alcançar uma formulação com alto teor de sólidos é o conhecimento da evolução da viscosidade com o aumento do teor de sólidos ${ }^{[9]}$. É importante salientar que o processo com alto teor de sólidos é uma condição limite de estabilidade coloidal e, próximo deste limite, alguma perturbação no processo, mesmo pequena, poderá provocar o rápido aumento da viscosidade, restando dentro do reator uma pasta, provocando perda do produto e parada para limpeza.

A viscosidade de uma emulsão é função da concentração de sólidos, da Distribuição de Tamanho de Partículas (DTP) e do diâmetro médio das mesmas ${ }^{[8,9]}$, além da ação de aditivos, como protetores coloidais (por exemplo, poli (álcoolvinílico) (PAV), hidroxietilcelulose (HEC), poli (etileno glicol) (PEG)) e estabilizantes. De um modo geral a viscosidade diminui com o aumento do tamanho médio das partículas, com o alargamento e o com o número de modas da DTP. A presença de aditivos aumenta a viscosidade da emulsão.

As viscosidades das emulsões com teor de sólidos menor que $55 \%(\mathrm{~m} / \mathrm{m})$ são, usualmente, baixas, exceto para DTP monodispersa, visto que, para esta condição, as emulsões podem ter alta viscosidade. Para teores de sólidos superiores a $55 \%(\mathrm{~m} / \mathrm{m})$ a viscosidade aumenta rapidamente e é muito dependente da taxa de cisalhamento. Esta característica tem implicações no projeto do reator (como por exemplo, agitação e transferência de calor), bombeamento, transferência e manuseio da emulsão.

Existe um limite físico à compactação das partículas no meio; ele é de $74 \%(\mathrm{~m} / \mathrm{m})$ para o caso de todas as partículas serem esféricas de tamanhos uniformes ordenadas segundo uma malha de face centrada e em contato. No caso dos látices este limite superior é muito menor, porque as partículas não podem repousar em um estado ordenado devido à agitação e porque elas não podem entrar em contato sem serem desestabilizadas.

Um dos látices de maior importância industrial é o do copolímero Acetato de Vinila/Acrilato de Butila (AV/AB) com fração mássica de $\mathrm{AB}$ de $15-25 \%(\mathrm{~m} / \mathrm{m})^{[10]}$, que é amplamente utilizada na composição de tintas. Este sistema é caracterizado por grandes diferenças de razões de reatividade $\left(r_{A V}=0,05\right.$ e $\left.r_{A B}=5,5\right)$, taxas de solubilidade em água $\left(25 \mathrm{~g} / \mathrm{L}\right.$ para o AV e $1-1,4 \mathrm{~g} / \mathrm{L}$ para o $\mathrm{AB}$, a $\left.25^{\circ} \mathrm{C}\right)$, constantes da taxa de propagação $\left(k_{P}(\mathrm{AV})=4000 \mathrm{~L} / \mathrm{mol}\right.$.s e $k_{P}(\mathrm{AB})=200 \mathrm{~L} / \mathrm{mol} . \mathrm{s}$, a $60{ }^{\circ} \mathrm{C}$ ) e de temperatura de transição vítrea, $T_{g}\left(T_{g}(\mathrm{AV})=32{ }^{\circ} \mathrm{C}\right.$ e $\left.T_{g}(\mathrm{AB})=-54^{\circ} \mathrm{C}\right)$. Devido a estas diferenças significativas nas propriedades dos monômeros, os látices resultantes podem ter diferenças significativas de propriedades morfológicas e de partículas dependendo da composição do copolímero, que também influencia a TMFF das emulsões e a $T_{g}$ dos polímeros.

A polimerização em emulsão pode ser realizada em diferentes tipos de reatores (batelada, semibatelada ou contínuo), porém, nenhum é tão versátil quanto o reator semibatelada, embora, não seja tão utilizado quanto os outros tipos de reatore ${ }^{[11]}$. A principal característica do processo de polimerização em reator semibatelada é a sua maior flexibilidade operacional em relação aos outros processos. Nos reatores semibatelada pode-se manipular independentemente diversas variáveis, como: alimentação de monômeros (puros, em solução ou emulsionados), iniciadores, emulsificantes, protetor coloidal e aditivos; agitação (velocidade e tipo de agitador); temperatura (fixa ou variável). Ainda são muito importantes a quantidade e a composição da carga inicial no reator.

Para a estabilização das partículas utilizam-se, usualmente, emulsificantes iônicos (por exemplo, dodecilsulfato de sódio), porém, estes emulsificantes favorecem a formação de partículas pequenas, da ordem de 120-130 $\mathrm{nm}^{[4]}$. Além disso, estes emulsificantes não são muito efetivos para teor de sólidos superior a $40 \%(\mathrm{~m} / \mathrm{m})$, devido ao excesso de eletrólitos.

Partículas maiores podem ser obtidas com emulsificantes não iônicos (por exemplo, alquilbenzenoetoxilados), porém, estes emulsificantes favorecem a nucleação homogênea e a coagulação das partículas, mesmo para concentrações da ordem de 40 vezes a concentração micelar crítica $(\mathrm{CMC})$. No entanto, uma mistura de emulsificantes não-iônico e iônico permite uma melhor estabilização das partículas com menor taxa de coagulação ${ }^{[4]}$. Por outro lado, aqueles autores relataram que o processo semibatelada com baixa vazão de alimentação dos monômeros favorece a estabilidade do látex.

O AV tem caráter hidrofílico, o que dificulta a adsorção do emulsificante sobre as partículas e a estabilidade coloidal. Devido a este caráter peculiar das emulsões vinil-acrílicas há necessidade de outros métodos de estabilização suplementares como, monômeros funcionais (por exemplo, ácido acrílico), protetores coloidais, que estabilizam as partículas estereamente ${ }^{[12]}$ e emulsificantes polimerizáveis ${ }^{[13]}$.

O objetivo do presente trabalho é obter emulsões vinilacrílicas com alto teor de sólidos investigando a influência da concentração de uma mistura de emulsificantes iônico (sulfato sódico de nonilfenol etoxilado) e não iônico (nonilfenol etoxilado) e da concentração de protetor coloidal, poli(álcool vinílico), sobre a cinética de polimerização, teor máximo de polímeros, a estabilidade do látex, o tamanho médio de partículas e a viscosidade das emulsões obtidas.

\section{Experimental}

\section{Reagentes}

Os produtos químicos utilizados, de grau comercial, foram: acetato de vinila (contendo $12 \mathrm{ppm}(\mathrm{mol} / \mathrm{mol})$ de inibidor hidroquinona), acrilato de butila (contendo $60 \mathrm{ppm}$ (mol/mol) de inibidor), de grau comercial, fornecidos pela 
Rhône Poulenc e utilizados conforme recebidos. Nonilfenol etoxilado com 23 mols de óxido de etileno $\left(\right.$ RENEX $^{\circledR} 230$ fornecido pela Oxiteno SA) e sulfato sódico de nonilfenol etoxilado com 25 mols de óxido de etileno (DISPONIL ${ }^{\circledR} 25 \mathrm{~S}$ fornecido pela Cognis SA), emulsificantes não iônico e iônico, respectivamente, e poli (álcool vinílico) (PVA ERKOL ${ }^{\circledR}$ 5/88 fornecido pela Erkol SA), protetor coloidal. Os monômeros não foram purificados porque se visa à obtenção de resultados aplicáveis a procedimentos industriais, nos quais os monômeros contêm inibidor e não é feita desoxigenação.

Os produtos químicos utilizados, de grau de laboratório, foram: solução aquosa de hidroperóxido de t-butila a $70 \%$ $(\mathrm{m} / \mathrm{m})$ (TRIGONOX ${ }^{\circledR}$ A-W70 fornecido pela Akzo Nobel $\mathrm{SA})$ e sulfoxilato de sódio formaldeído $\left(\right.$ COSMOLIT $^{\circledR}$ fornecido pela Cosmoquímica SA) (iniciadores), bicarbonato de sódio (regulador de pH), n-dodecil mercaptana (agente de transferência de cadeia) (nDDM) e hidroquinona, usada para inibir a reação. O bicarbonato de sódio, nDDM e hidroquinona foram fornecidos pela Labsynth Ltda. Utilizou-se água deionizada em todos os ensaios.

A CMC do emulsificante não-iônico, $0,34 \mathrm{~g} / \mathrm{L}$, e do iônico, $0,40 \mathrm{~g} / \mathrm{L}$, a $25^{\circ} \mathrm{C}$, foram estimadas usando correlações baseadas na literatura ${ }^{[14]}$.

A formulação básica de polimerização para teor total de sólidos $69 \%(\mathrm{~m} / \mathrm{m})$ está mostrada na Tabela 1 .

As concentrações de emulsificantes, de protetor coloidal, de bicarbonato de sódio, de nDDM e de iniciadores foram aumentadas proporcionalmente à concentração de monômeros, para teores de sólidos 71 e $73 \%(\mathrm{~m} / \mathrm{m})$

Foram testadas 3 concentrações de emulsificantes, [E], e 3 concentrações de protetor coloidal, [P], definidas em termos de $\%(\mathrm{~m} / \mathrm{m})$ em relação à carga total alimentada ao reator. As concentrações de emulsificantes utilizadas foram de 1,0, 1,2 e 1,4 vezes à concentração da formulação básica.
As concentrações de protetores coloidais utilizadas foram de 1,0, 2,0 e 3,0 vezes à concentração da formulação básica.

\section{Processo de polimerização}

Os ensaios foram realizados em um reator de vidro de $1 \mathrm{~L}$ de capacidade, encamisado para aquecimento ou resfriamento com água. O reator dispõe de agitador com indicação de rotação e torque constante, entrada para desoxigenação do meio reativo com nitrogênio, saída para condensador, controle de temperatura com precisão de $\pm 0,5^{\circ} \mathrm{C}$, entradas para alimentação simultânea e independente de pré-emulsão (com bomba de diafragma), de iniciadores [soluções aquosas de sulfoxilato de sódio formaldeído e de hidroperóxido de t-butila (com bomba peristáltica dupla)] e válvula de fundo para amostragem.

A carga de pré-emulsão alimentada foi monitorada ao longo do tempo através da indicação de balança eletrônica, sobre a qual se encontrava o recipiente com a pré-emulsão preparada anteriormente.

$\mathrm{O}$ procedimento experimental consistiu em introduzir inicialmente no reator $43 \%(\mathrm{~m} / \mathrm{m})$ da água, $80 \%(\mathrm{~m} / \mathrm{m})$ dos emulsificantes e $100 \%(\mathrm{~m} / \mathrm{m})$ do protetor coloidal e do regulador de $\mathrm{pH}$. A carga inicial era então desoxigenada com nitrogênio por $1 \mathrm{~h}$ e aquecida à temperatura de $74{ }^{\circ} \mathrm{C}$.

$\mathrm{O}$ ensaio iniciou-se com a alimentação da pré-emulsão e dos iniciadores. Neste instante o set-point da temperatura do reator foi aumentado para $90{ }^{\circ} \mathrm{C}$.

Durante o tempo total de alimentação, que foi de $3 \mathrm{~h}$ para todos os ensaios, a vazão de alimentação da pré-emulsão foi mantida constante. Os iniciadores foram alimentados por $3,5 \mathrm{~h}$ com vazão constante. Após o término da alimentação dos iniciadores, a reação prosseguiu por mais 30 min para garantir altas conversões e o consumo total dos monômeros, totalizando $4 \mathrm{~h}$ de reação.

Tabela 1. Formulação Básica de Polimerização.

\begin{tabular}{|c|c|c|c|}
\hline \multicolumn{4}{|c|}{ Reator } \\
\hline Reagente & $\mathrm{TS}=69 \%(\mathrm{~m} / \mathrm{m})$ & $\mathrm{TS}=71 \%(\mathrm{~m} / \mathrm{m})$ & $\mathrm{TS}=73 \%(\mathrm{~m} / \mathrm{m})$ \\
\hline Água & 15,6 & 13,5 & 11,4 \\
\hline Nonil Fenol Etoxilado & 2,58 & 2,66 & 2,74 \\
\hline Sulfato Sódico de Nonilfenol Etoxilado & 0,504 & 0,517 & 0,535 \\
\hline PAV & 0,277 & 0,286 & 0,294 \\
\hline Bicarbonato de Sódio & 0,324 & 0,334 & 0,343 \\
\hline \multicolumn{4}{|c|}{ Pré-emulsão } \\
\hline Acetato de Vinila & 51,7 & 53,3 & 54,8 \\
\hline Acrilato de Butila & 12,9 & 13,3 & 13,7 \\
\hline Nonil Fenol Etoxilado & 0,462 & 0,477 & 0,490 \\
\hline Sulfato Sódico de Nonilfenol Etoxilado & 0,0900 & 0,0926 & 0,0952 \\
\hline Água & 6,52 & 6,52 & 6,52 \\
\hline n-dodecil mercaptana (nDDM) & 0,125 & 0,129 & 0,133 \\
\hline \multicolumn{4}{|c|}{ Iniciadores } \\
\hline Sulfoxilato de Sódio Formaldeído & 4,40 & 4,40 & 4,40 \\
\hline Hidroperóxido de t-butila [sol. aq. $70 \%$ (v/v)] & 4,40 & 4,40 & 4,40 \\
\hline
\end{tabular}




\section{Métodos Analíticos}

A fração de sólidos totais no látex foi determinada por gravimetria em estufa a $90{ }^{\circ} \mathrm{C}$, na qual as amostras foram deixadas por $12 \mathrm{~h}$.

As amostras, todas com cerca de $1 \mathrm{~g}$, foram retiradas do reator em intervalos de $5 \mathrm{~min}$, até um tempo de reação de 30 min, e a cada 10 min, até o final da reação. As amostras foram colhidas em placas metálicas contendo cerca de $0,4 \mathrm{~g}$ de solução aquosa de hidroquinona a $1 \%(\mathrm{~m} / \mathrm{m})$. As amostragens e pesagens foram feitas em menos de 30 segundos.

A fração de polímeros na amostra foi determinada subtraindo-se da fração de sólidos totais, a fração de sólidos que não participam da reação de polimerização (emulsificantes, protetor coloidal, bicarbonato de sódio, iniciadores e agente de transferência de cadeia), adicionado ao reator até o instante $\mathrm{t}$ da respectiva amostragem.

A conversão global dos monômeros foi determinada através da relação entre a fração de polímeros e a massa total de monômeros na formulação de polimerização.

O tamanho médio das partículas foi determinado por Espectroscopia de Correlação de Fótons (PCS) com um aparelho Coulter N4 PLUS. Este aparelho permite analisar partículas com uma faixa de tamanhos de 3 a $3000 \mathrm{~nm}$, equipado com lâmpada laser He-Ne de $10 \mathrm{~mW}$, de comprimento de onda $632,8 \mathrm{~nm}$, e correlacionador digital de 80 canais.

As amostras, de cerca de $1 \mathrm{~g}$ cada uma, foram colhidas em frascos de $5 \mathrm{~mL}$ com tampa e batoque contendo $2 \mathrm{~mL}$ de água deionizada, 1 gota de solução aquosa de hidroquinona $1 \%(\mathrm{~m} / \mathrm{m})$ e 10 gotas de solução aquosa de emulsificante a $10 \%(\mathrm{~m} / \mathrm{m})$. As amostras, cerca de $0,01 \mathrm{~mL}$, foram então diluídas em água deionizada em cubetas de poliestireno de $3 \mathrm{~cm}^{3}$. Com a diluição em água as partículas perdem os monômeros residuais e a análise resulta no tamanho das partículas não-inchadas ${ }^{[15]}$.

Os erros experimentais nas medidas de conversão global e de tamanho médio de partículas foram inferiores a $\pm 5 \%$.

A viscosidade aparente dos látices sintetizados foi determinada com viscosímetro Brookfield RVDV-III com haste $\mathrm{SC} 4-21$ a $20{ }^{\circ} \mathrm{C}$.

\section{Resultados e Discussão}

\section{Influência da concentração de protetor coloidal e de emulsificantes}

A Figura 1 mostra os resultados de teor de sólidos, tamanho médio de partículas e de viscosidade aparente, para algumas concentrações de protetor coloidal e de emulsificantes.

A análise da Figura 1a mostra que há uma pequena influência da concentração de protetor coloidal e de emulsificantes sobre o teor de sólidos. Observa-se que o teor de sólido é pouco influenciado pela variação de concentração de protetor coloidal e de emulsificantes, provavelmente devido à alta concentração de iniciadores utilizada.
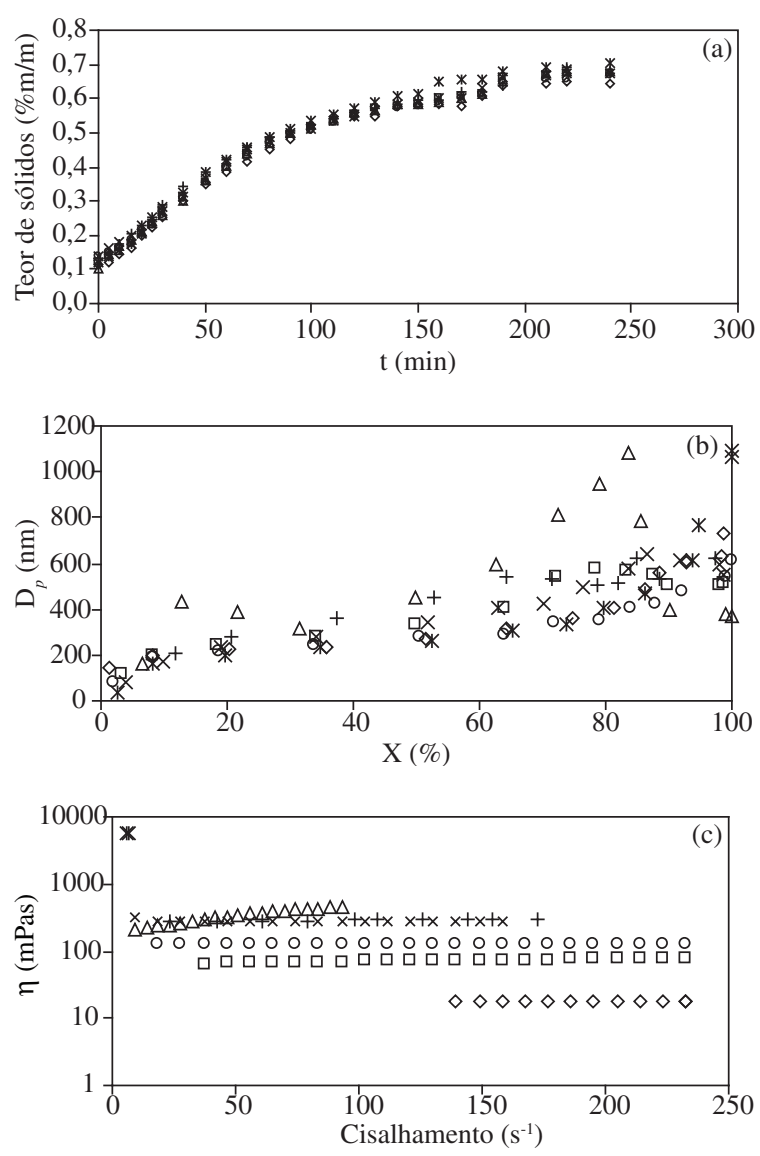

\begin{tabular}{ccccc}
\hline & {$[\mathbf{P}]$} & {$[\mathbf{E}]$} & TS & TP \\
\cline { 2 - 5 } & \multicolumn{4}{c}{$\%(\mathbf{m} / \mathbf{m})$} \\
$\square$ & 0,093 & 2,79 & 64,6 & 60,6 \\
$\Delta$ & 0,093 & 3,23 & 67,1 & 62,7 \\
+ & 0,093 & 3,50 & 68,2 & 63,4 \\
0 & 0,186 & 2,78 & 67,6 & 63,5 \\
ж & 0,185 & 3,22 & 66,5 & 61,9 \\
$\times$ & 0,279 & 2,79 & 70,1 & 65,8 \\
\hline
\end{tabular}

Figura 1. Influência da concentração de protetor coloidal e de emulsificantes sobre (a) teor de sólidos, (b) tamanho médio de partículas, $D_{p}$ e (c) viscosidade aparente, $\eta$ a $T=20^{\circ} \mathrm{C}$. (Teor total de sólidos na formulação básica $=69 \%(\mathrm{~m} / \mathrm{m}) ; X=$ conversão total $; T S, T P=$ teor total final de sólidos e de polímero em relação à carga total alimentada ao reator; $[\mathrm{P}],[\mathrm{E}]=$ teor total de protetor coloidal e de emulsificantes em relação à carga total alimentada ao reator).

Com o término da alimentação de pré-emulsão, aos $180 \mathrm{~min}$, ocorre um salto de teor de sólidos devido ao aumento da temperatura de cerca de 87 para $90{ }^{\circ} \mathrm{C}$.

Todas as reações foram realizadas em condições nas quais a taxa de polimerização superava a de alimentação de monômeros (starved condition). Esta condição foi constatada pelos resultados de conversões instantâneas, não mostradas, superiores a $90 \%$ desde os primeiros 5 min de reação.

A análise da Figura $1 b$ mostra que a evolução dos tamanhos médios com a conversão foi semelhante nos diferentes 
ensaios, variando entre 500 e $600 \mathrm{~nm}$, exceto para os ensaios com $[\mathrm{P}]=0,093$ e $[\mathrm{E}]=3,50 \%(\mathrm{~m} / \mathrm{m})$ e $[\mathrm{P}]=0,279$ e $[\mathrm{E}]=2,79 \%(\mathrm{~m} / \mathrm{m})$. Nestes casos as emulsões apresentaram partículas com tamanhos médios próximos de $1000 \mathrm{~nm}$ e, portanto, menos estáveis, ao se considerar que as partículas maiores correspondem a látices menos estáveis. Excesso de emulsificante desestabiliza a emulsão, provavelmente, devido ao aumento da afinidade das moléculas de emulsificante, aproximando as partículas e provocando coagulação.

Conforme comentado anteriormente, a distribuição de tamanhos de partículas (DTP) é um parâmetro muito importante que influencia a viscosidade da látice final. Porém, podemos analisar, em princípio, as viscosidades com base apenas no diâmetro médio das partículas, visto que a formulação e o processo são praticamente idênticos para todos os ensaios. Assim, as DTPs em todos os ensaios devem ser semelhantes, influenciando da mesma maneira as viscosidades finais das emulsões.

Parece haver uma relação das concentrações de protetor coloidal, [P], e de emulsificante, [E], para que haja estabilidade adequada das partículas. Uma relação muito baixa, ou muito alta, desestabiliza as partículas. Excesso de protetor coloidal leva a um mecanismo de desestabilização estérea, talvez por neutralização das cargas da partícula, "bridging" ou emaranhamento.

A Figura 1c mostra os resultados de viscosidade aparente dos látices finais. Observa-se que o comportamento de todos os látices sintetizados é praticamente Newtoniano, existe forte interação entre as partículas (altas viscosidades), não há interstícios vazios entre elas (o comportamento seria dilatante se houvessem interstícios vazios) e nem alinhamento das partículas ou deformação da dupla camada elétrica (o comportamento seria pseudoplástico).

Observa-se que a viscosidade cresce com a concentração de emulsificante para $[\mathrm{P}]=0,093 \%(\mathrm{~m} / \mathrm{m})$; depende pouco da concentração de emulsificantes para $[\mathrm{P}]=0,186 \%(\mathrm{~m} / \mathrm{m})$ e cai com a concentração de emulsificantes para $[\mathrm{P}]=0,276 \%$ $(\mathrm{m} / \mathrm{m})$.

A viscosidade cresce com a concentração de protetor coloidal para $[\mathrm{E}]=2,79$ e $3,50 \%(\mathrm{~m} / \mathrm{m})$ e depende pouco da concentração de protetor coloidal para $[\mathrm{E}]=3,23 \%(\mathrm{~m} / \mathrm{m})$.

Para valores intermediários de concentração de protetor coloidal e de emulsificantes as viscosidades não variam muito, indicando que as interações entre as partículas são menos intensas e que as emulsões são mais estáveis.

Com base nos resultados obtidos para teor de sólidos da formulação de $69 \%(\mathrm{~m} / \mathrm{m})$, planejou-se uma nova série de experimentos para teor de sólidos na formulação de $71 \%$ $(\mathrm{m} / \mathrm{m})$. Para esta série manteve-se a proporcionalidade entre as concentrações de protetor coloidal e de emulsificantes igual à da formulação básica. Foram realizados 3 ensaios com as seguintes concentrações de protetor coloidal e de emulsificante: $[\mathrm{P}]=0,098 \mathrm{e}[\mathrm{E}]=2,88 \%(\mathrm{~m} / \mathrm{m}) ;[\mathrm{P}]=0,192 \mathrm{e}$ $[\mathrm{E}]=3,32 \%(\mathrm{~m} / \mathrm{m}) ;[\mathrm{P}]=0,286 \mathrm{e}[\mathrm{E}]=3,77 \%(\mathrm{~m} / \mathrm{m})$.
O ensaio com $[\mathrm{P}]=0,098$ e $[\mathrm{E}]=2,88 \%(\mathrm{~m} / \mathrm{m})$ coagulou antes do término da reação, indicando que com o aumento do teor de sólidos é necessário uma quantidade relativamente maior de protetor coloidal e de emulsificante para obter um látex estável.

A maior proximidade das partículas implica em maior interação entre as mesmas. A estabilidade coloidal é obtida com uma maior concentração de moléculas de emulsificantes e de protetor coloidal sobre a superfície das partículas.

A Figura 2 mostra os resultados para os ensaios com as seguintes concentrações de protetor coloidal e emulsificante: $[\mathrm{P}]=0,192$ e $[\mathrm{E}]=3,32 \%(\mathrm{~m} / \mathrm{m}) ;[\mathrm{P}]=0,286$ e $[\mathrm{E}]=3,37 \%$ $(\mathrm{m} / \mathrm{m})$.

A Figura 2a mostra que a evolução do teor de sólidos é pouco influenciada pela concentração de protetor coloidal e
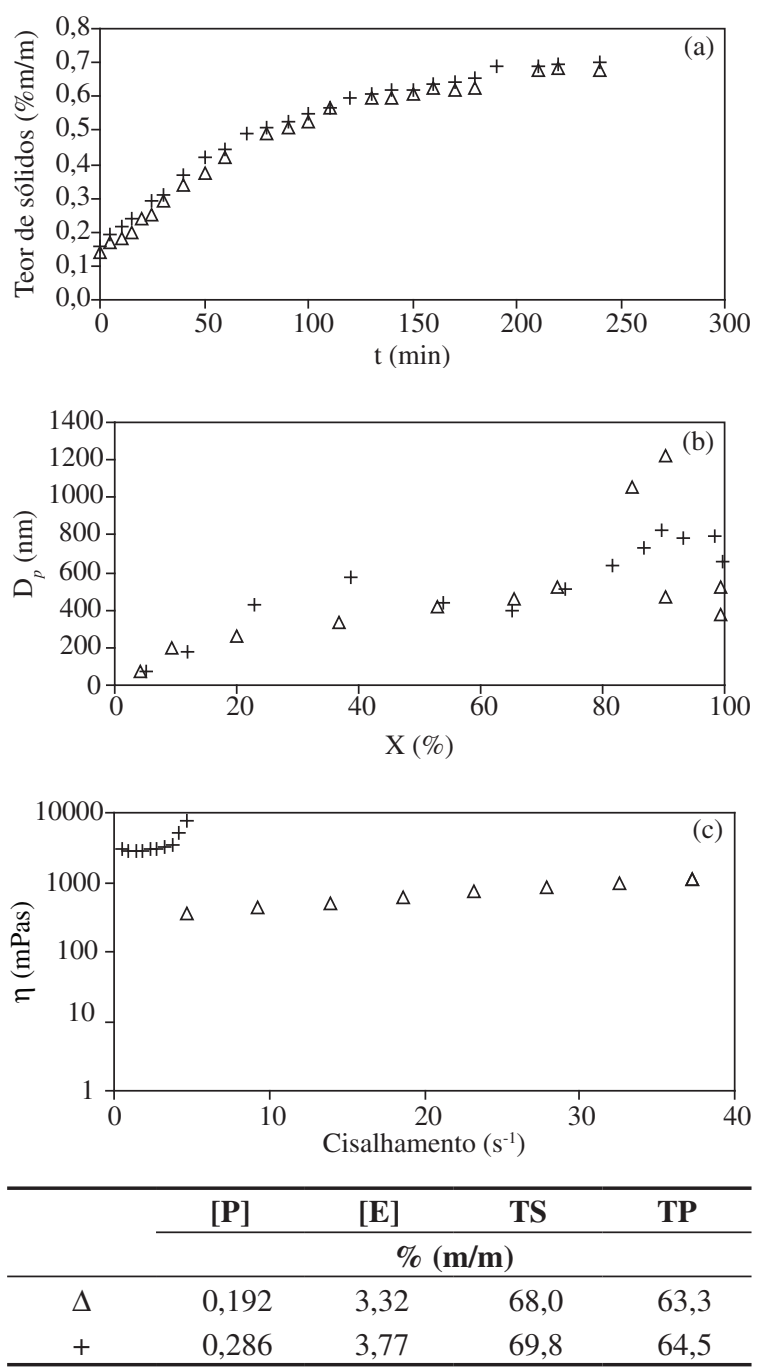

Figura 2. Influência da concentração de protetor coloidal e de emulsificantes sobre (a) teor de sólidos; (b) tamanho médio de partículas, $D_{p}$ e (c) viscosidade aparente, $\eta$ a $T=20{ }^{\circ} \mathrm{C}$. (Teor total de sólidos na formulação básica $=71 \%(\mathrm{~m} / \mathrm{m}) ; X=$ conversão total; $T S$, $T P=$ teor total final de sólidos e de polímero em relação à carga total alimentada ao reator; $[\mathrm{P}],[\mathrm{E}]=$ teor total de protetor coloidal e de emulsificantes em relação à carga total alimentada ao reator). 
de emulsificante. Os valores de teor de sólidos, ligeiramente maiores, devem-se justamente a esta maior concentração de sais e não de polímeros.

Como nos ensaios para teor de sólidos na formulação de $69 \%(\mathrm{~m} / \mathrm{m})$, Figura 1a, também se observa na Figura 2a um salto no teor de sólidos aos $180 \mathrm{~min}$ de reação, o que coincide com o término da alimentação de pré-emulsão.

A Figura $2 b$ mostra que as partículas são mais estáveis para as maiores concentrações de protetor coloidal e de emulsificantes. Para concentrações menores ocorre uma queda abrupta do tamanho médio das partículas, para um valor de conversão global de cerca de $90 \%$. Isto ocorre devido à coagulação das partículas maiores e separação de fases dentro do reator. O soro resultante ainda contém partículas parcialmente estáveis, porém menores.

A Figura 2c mostra que o comportamento dilatante (aumento de $\eta$ com o cisalhamento) dos látices é mais pronunciado com o aumento da concentração de protetor coloidal e de emulsificantes. Com o aumento da concentração de protetor coloidal e de emulsificantes a interação superficial entre as partículas aumenta e assim também a viscosidade e a estabilidade coloidal verificada anteriormente.

\section{Influência do teor de sólidos}

Com base nos resultados mostrados na Figura 2, constatou-se que o látex com teor de sólidos $71 \%(\mathrm{~m} / \mathrm{m})$ e nível intermediário de concentração de protetor coloidal e emulsificantes é instável. Por isto, planejou-se mais um ensaio com teor de sólidos igual a $73 \%(\mathrm{~m} / \mathrm{m})$ e maior concentração de protetor coloidal e emulsificantes: $[\mathrm{P}]=0,293 \mathrm{e}$ $[\mathrm{E}]=3,86 \%(\mathrm{~m} / \mathrm{m})$.

Estes resultados estão mostrados na Figura 3, juntamente com aqueles para teor de sólidos 69 e $71 \%(\mathrm{~m} / \mathrm{m})$, para as mesmas concentrações relativas de protetor coloidal e emulsificantes. A Figura 3a mostra que a evolução do teor de sólidos é pouco influenciada pelo teor de sólidos da formulação e também, como nas Figuras 1a e 2a, observa-se um salto no teor de sólidos aos 180 min de reação com o término da alimentação de pré-emulsão.

A Figura $3 b$ mostra que as partículas são estáveis para os 3 teores de sólido estudados e, dentro das imprecisões experimentais, os tamanhos médios durante a reação são semelhantes.

Os tamanhos médios das partículas para os resultados com teor de sólidos $73 \%(\mathrm{~m} / \mathrm{m})$ são maiores no final da reação. Isto se deve a erros experimentais decorrentes das dificuldades na amostragem da emulsão com alto teor de sólidos, devido a sua alta viscosidade e secagem rápida.

A Figura $3 \mathrm{c}$ mostra que a viscosidade cresce com o teor de sólidos, o comportamento da emulsão para teor de sólidos $69 \%(\mathrm{~m} / \mathrm{m})$ é Newtoniano, e que para teor de sólidos $71 \%$ $(\mathrm{m} / \mathrm{m})$ o comportamento é dilatante.

Devido à limitação do reômetro foi possível, para teor de sólidos $73 \%(\mathrm{~m} / \mathrm{m})$, obter apenas um valor de viscosidade de $365.000 \mathrm{cP}$ a uma taxa de cisalhamento de $0,07 \mathrm{~s}^{-1}$, a $20^{\circ} \mathrm{C}$.
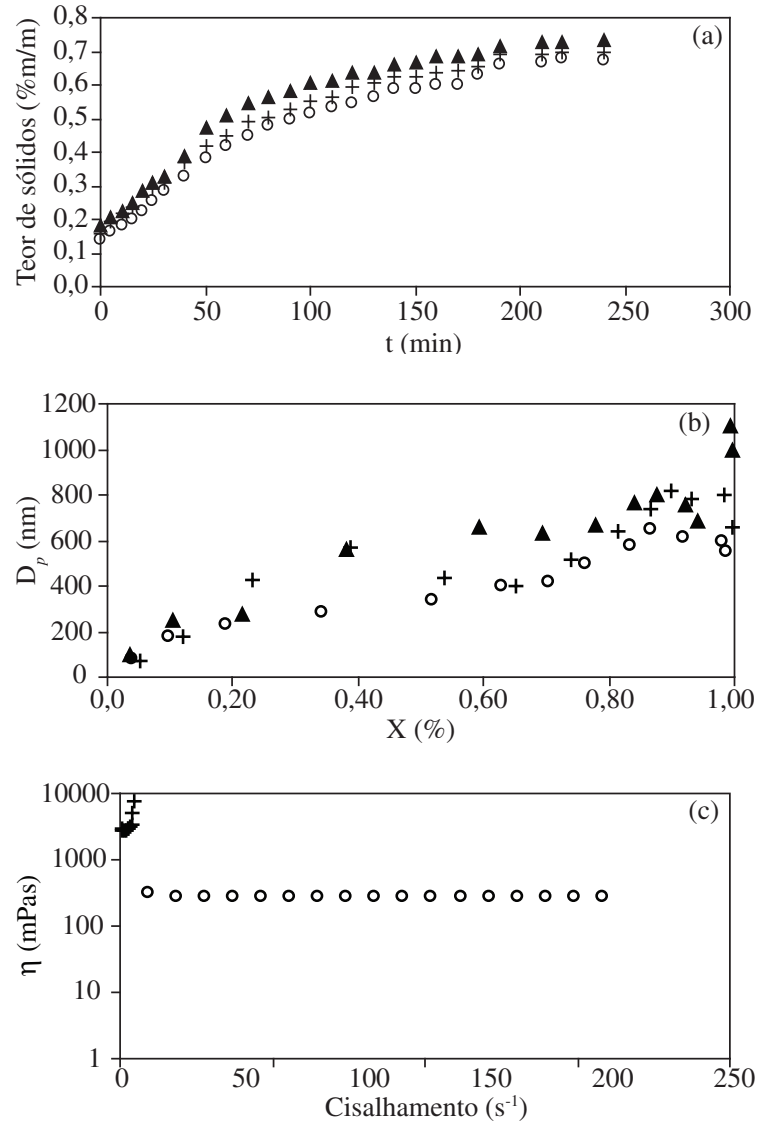

\begin{tabular}{lllll}
\hline & {$[\mathbf{P}]$} & {$[\mathbf{E}]$} & $\mathbf{T S}$ & $\mathbf{T P}$ \\
\cline { 2 - 5 } & \multicolumn{4}{c}{$\boldsymbol{\%}(\mathbf{m} / \mathbf{m})$} \\
\hline $\mathrm{o}$ & 0,277 & 3,64 & 67,2 & 62,1 \\
+ & 0,286 & 3,77 & 69,8 & 64,5 \\
$\boldsymbol{\Delta}$ & 0,293 & 3,86 & 73,2 & 67,7 \\
\hline
\end{tabular}

Figura 3. Influência da concentração de protetor coloidal e de emulsificantes sobre (a) teor de sólidos, (b) tamanho médio de partículas, $D_{p}$ e (c) viscosidade aparente, $\eta$ a $T=20^{\circ} \mathrm{C}$. (Teor total de sólidos na formulação básica $=$ $73 \%(\mathrm{~m} / \mathrm{m}) ; X=$ conversão total; $T S, T P=$ teor total final de sólidos e de polímero em relação à carga total alimentada ao reator; $[\mathrm{P}],[\mathrm{E}]=$ teor total de protetor coloidal e de emulsificantes em relação à carga total alimentada ao reator).

\section{Conclusões}

Dos ensaios realizados concluiu-se que a concentração de protetor coloidal e de emulsificantes não influenciou o teor de sólidos e a evolução da conversão. Verificou-se que para a relação mássica entre as concentrações de emulsificantes e de protetor coloidal $[\mathrm{E}] /[\mathrm{P}] \approx 13$ a emulsão é mais estável, o que corresponde ao menor tamanho médio de partículas. $\mathrm{O}$ comportamento reológico das emulsões é Newtoniano para todas as emulsões, exceto para altas concentrações de protetor coloidal e teor de sólidos de 71 e $73 \%(\mathrm{~m} / \mathrm{m})$. Nestas condições o comportamento é dilatante. A viscosidade cresce com o teor de sólidos e com a concentração de protetor coloidal. Os resultados do presente trabalho podem auxiliar 
no desenvolvimento de processos de obtenção de látices com altos teores de sólidos para outros sistemas poliméricos.

\section{Referências Bibliográficas}

1. Masa, J. A; Arbina, L. L. \& Asua, J. M. - A comparison between miniemulsion and conventional emulsion terpolymerization of styrene, 2-ethylhexyl acrilate and methacrylic acid. Journal of Applied Polymer Science, 48, p. 2055 (1993).

2. Tang, C. \& Chu, F. - Semicontinuous emulsion polymerization of styrene-butyl acrylate-methacrylic acid with high solid content. Journal of Applied Polymer Science, 82, p. 2352 (2001).

3. Schneider, M.; Graillat, C.; Guyot, A. \& McKenna, T. F. - High solids content emulsions. Part III. Synthesis of concentrated latexes via classic emulsion polymerization. Journal of Applied Polymer Science, 84, p. 1916 (2002).

4. Schneider, M.; Graillat, C.; Guyot, A. \& McKenna, T. F. - High solids content emulsions. Part II. Preparation of seed latexes. Journal of Applied Polymer Science, 84, p. 1897 (2002).

5. Do Amaral, M. \& Asua, J. M. - Synthesis of high solidcontent latex using alkali-soluble resin as sole surfactant, Macromolecular Rapid Communications, 25, p. 1883 (2004).

6. Do Amaral, M. \& Asua, J. M. - Synthesis of large, high solid-content latexes by miniemulsion polymerization. Journal of Polymer Science Part A - Polymer Chemistry, 42, p. 4222 (2004).

7. Boutti, S.; Graillat, C. \& McKenna, T. F. - New routes to high solid content latexes: a process for in situ particle nucleation and growth. Macromolecular Symposia, 206, p. 383 (2004).
8. Do Amaral, M.; Van Es, S. \& Asua, J. M. - Effect of the particle size distribution on the low shear viscosity of high-solid-content latexes. Journal of Polymer Science Part A - Polymer Chemistry, 42, p. 3936 (2004).

9. Schneider, M.; Claverie, J.; Graillat, C. \& McKenna, T. F. - High solids content emulsions. Part I. A study of the influence of the particle size distribution and polymer concentration on viscosity. Journal of Applied Polymer Science, 84, p. 1878 (2002).

10. Lazaridis, N.; Alexopoulos, A. H. \& Kiparissides, C. Semi-batch emulsion copolymerization of vinyl acetate and butyl acrylate using oligomeric nonionic surfactants. Macromolecular Chemistry and Physics, 202, p.2614 (2001).

11. Li, B. \& Brooks, B. W. - Semi-batch processes for emulsion polymerization. Polymer International, 29, p. 41 (1992).

12. Westby, M. J. - Non-ionic emulsion polymerization. Colloid Polym. Sci., 266, p. 46 (1988).

13. Uzulina, I.; Zicmanis, A.; Graillat, C.; Claverie, J. \& Guyot, A. - Nonionic maleic surfmers. Journal of Dispersion Science and Technology, 23, p. 799 (2002).

14. Huibers, D. T.; Lobanov, V. S.; Katritzky, A. R.; Shah, D. O. \& Karelson, M. - Prediction of critical micelle concentration using a quantitative structure-property relationship approach. 2. Anionic surfactants,. Journal of Colloid and Interface Science, 187, p. 120 (1997).

15. Araújo, P. H. H. - "Distribuição de tamanhos de partícula em sistemas heterogêneos de polimerização", Tese de Doutorado, Universidade Federal do Rio de Janeiro, Brasil (1999).

Enviado: 23/01/06

Reenviado: 19/07/06

Aceito: 04/08/06 